ARTICLE

DOI: $10.1038 / \mathrm{s} 41467-018-05919-6$

\title{
Dye-incorporated coordination polymers for direct photocatalytic trifluoromethylation of aromatics at metabolically susceptible positions
}

Tiexin Zhang ${ }^{1}$, Xiangyang Guo ${ }^{1}$, Yusheng Shi ${ }^{1}$, Cheng $\mathrm{He}^{1} \&$ Chunying Duan (i) ${ }^{1,2}$

Direct trifluoromethylation of unactivated aromatic rings at metabolically susceptible positions is highly desirable in pharmaceutical applications. By incorporating thiophenes into the backbone of triphenylamine to enlarge its $\pi$-system, a new approach for constructing coordination polymers is reported for direct trifluoromethylation without prefunctionalization of the aryl precursors. The improved light-harvesting ability and well-modulated excited state redox potential of the designed polymers endow the generated $\mathrm{CF}_{3}$ radicals with suitable reactivity and enhance radical adduct oxidation in pores. The well-configurated interactions between the organic ligands distort the coordination geometry to create active interaction sites within the coordination polymer; thus, the substrates could be docked near the photoredox-active centres. The synergistic electronic and spatial effects in the confined pores balance the contradictory demands of electronic effects and reaction dynamics, achieving regio- and diastereoselective discrimination among reaction sites with unremarkable electronic/steric differences.

\footnotetext{
${ }^{1}$ State Key Laboratory of Fine Chemicals, Dalian University of Technology, Dalian 116024 , China. ${ }^{2}$ Collaborative Innovation Center of Chemical Science and Engineering, Tianjin 300071, China. Correspondence and requests for materials should be addressed to C.D. (email: cyduan@dlut.edu.cn)
} 
T he installation of a trifluoromethyl group onto metabolically susceptible positions on aromatic rings increases the robustness of the corresponding drug candidates towards oxidative metabolism in vivo ${ }^{1}$, making the trifluoromethylation of aromatics a highly desirable chemical reaction ${ }^{2,3}$. In light of the photoinduced generation of highly reactive $\mathrm{CF}_{3}$ radicals, polypyridyl noble metal complexes enable direct $\mathrm{C}-\mathrm{H}$ trifluoromethylation of unactivated aromatics under visible-light irradiation ${ }^{4}$. This approach bypasses tedious prefunctionalization of aryl precursors that is needed for transition metal-catalysed cross-couplings using a nucleophilic or electrophilic source of $\mathrm{CF}_{3}{ }^{5,6}$. However, the discrimination of various aromatic positions without distinct electronic contributions is thermodynamically difficult due to the high electrophilicity of $\mathrm{CF}_{3}$ radicals. Trifluoromethylation of drugs with aromatic cores can protect them from aryl oxidative metabolism by enzymes in living cells (i.e., cytochrome P450). Thus, appending trifluoromethyl groups to the metabolically susceptible positions of aromatics without prefunctionalization of precursors ${ }^{7}$ is highly desirable, and it is promising to achieve this goal by merging all the catalytic requirements to controllably generate $\mathrm{CF}_{3}$ radicals under visible light and trigger the metabolically susceptible positions of aromatic drug candidates.

Porous coordination polymers exhibit confined pores similar to those in molecular sieves ${ }^{8,9}$, and the tenability and flexibility of the material allow for uniform heterogenization of photoactive organic dyes at high densities around the pores ${ }^{10-14}$. As the integration of near-ultraviolet (UV) responsive triphenylamine (TPA) and covalently binding group L-proline derivative into a single coordination polymer has been proven to be an effective approach to the photocatalytic $\alpha_{-}{ }^{15}$ or $\beta$-functionalization ${ }^{16}$ of saturated carbonyl compounds, we believe that merging the TPAbased chromophore with modulated photoelectronic property and the noncovalently binding site together within one framework is a potential strategy for controllably generating $\mathrm{CF}_{3}$ radicals under visible-light irradiation and harnessing the aromatic drug candidates that lack of covalent binding moieties.

Here, we show that incorporating thiophene groups into the ligand ${ }^{14}$ bearing TPA moiety allows us to modify the potential photochemical properties of TPA-based coordination polymer with the aim of formulating a new system for direct trifluoromethylation of aromatics in a heterogeneous manner (Fig. 1). We envision that the enlarged $\pi$-system created by the insertion of thiophene moieties into the backbone of the TPAbased ligand would improve the visible-light harvesting ability and tune the photoelectronic performance to generate reactive $\mathrm{CF}_{3}$ radicals with milder reactivity, and allow for more efficient oxidation of the radical adduct. Simultaneously, enhanced interactions between organic dyes cause the metal nodes to distort, creating coordination vacancies for the docking of substrates in close proximity to the photocatalytically active centres (Fig. 1c). Furthermore, the intrinsic crystalline nature of coordination polymers allows for structural analyses of the intermediates formed in each activation and catalytic step, offering an excellent platform for studying photon capture, electron delivery, and catalytic activation ${ }^{17,18}$.

\section{Results}

Synthesis and characterization of the photocatalyst. A solvothermal reaction between $\mathrm{H}_{3}$ TCTA (tris[4-(5-carboxy-2-thienyl)phenyl]amine) and $\mathrm{Zn}\left(\mathrm{NO}_{3}\right)_{2} \cdot 6 \mathrm{H}_{2} \mathrm{O}$ in DMF at $100^{\circ} \mathrm{C}$ afforded a new coordination polymer, Zn-TCTA, in a $70 \%$ yield. Single-crystal X-ray structural analysis revealed that this polymer crystallizes in the $R-3 c$ space group. The distorted geometry of the $\mathrm{Zn}_{4} \mathrm{O}$ units is enforced by interlayer $\pi \cdots \pi$ interactions between
TCTA moieties, endowing zinc atoms with empty coordination sites for potential substrate binding and activation (Supplementary Fig. 4). Each TCTA ${ }^{3-}$ anion bridges three $\mathrm{Zn}_{4} \mathrm{O}$ clusters, and each $\mathrm{Zn}_{4} \mathrm{O}$ cluster is connected to six different TCTA ${ }^{3-}$ anions, forming a two-dimensional sheet. These deprotonated ligands are located above or below the two-dimensional sheets, creating undulating surfaces and intralayer cavities in the metal-organic sheets (Fig. 1b). Adjacent sheets assemble in an $\mathrm{ABCABC}$ fashion through $\pi \cdots \pi$ stacking interactions between the planar 4thiophenylphenyl moieties of the ligands to form a threedimensional porous structure (Fig. 1d). It is postulated that the interlayer stacking mode can enhance the excitation delocalization and light-harvesting ability of polymers ${ }^{19}$, benefiting photocatalytic reactions under visible-light irradiation. Additionally, the interlayer stacking mode is also expected to provide the possibility of exfoliating the polymers into two-dimensional materials ${ }^{20}$, which is expected to enhance their catalytic properties. The hexagonal pocket windows of the intralayer cavities were plugged from the direction of the $c$-axis to retain horizontal open channels with cross-sections of $10.4 \times 18.0 \AA^{2}$ (Fig. 1d). A methylene blue dye uptake experiment with $\mathrm{Zn}$-TCTA was used to demonstrate the guest accessibility, and the experiement yielded a dye uptake of $2.8 \%$ of the catalyst weight, as determined by the UV-vis spectra (Supplementary Fig. 8).

The UV-vis absorption spectrum of solid-state Zn-TCTA exhibited a remarkable redshift and broader absorption band than that observed for TPA-based MOF-150 ${ }^{14}$ (Fig. 2a and Supplementary Fig. 9). Solid-state electrochemical measurements revealed an oxidative potential of $1.17 \mathrm{~V}$ (Supplementary Fig. 10), and the reductive potential of the excited state was determined to be $-1.22 \mathrm{~V}$ on the basis of a free energy change of $2.39 \mathrm{eV}$ (Fig. 2a). In comparison to the photocatalyst $f a c-\operatorname{Ir}(\mathrm{Fppy})_{3}{ }^{21}, \mathrm{Zn}-$ TCTA has a weaker but sufficient photoreductive ability to reduce trifluoromethanesulfonyl chloride $\left(\mathrm{TfCl}, E_{1 / 2}{ }^{\mathrm{red}}=-0.18\right.$ V vs. saturated calomel electrode) ${ }^{4}$ and generate $\mathrm{CF}_{3}$ radicals with milder reactivity. The more positive oxidative potential of the dyes in $\mathrm{Zn}$-TCTA is expected to oxidize the $\mathrm{CF}_{3}$ radical adduct more efficiently to complete the catalytic cycle. The quenched emission at $546 \mathrm{~nm}$ (Fig. 2b) and the substantially decreased fluorescence lifetime (Supplementary Fig. 12) of the Zn-TCTA suspension upon the addition of $\mathrm{TfCl}$ indicated a classical photoinduced electron transfer process from the excited state of the TCTA moiety to $\mathrm{TfCl}$, which directly yielded active $\mathrm{CF}_{3}$ radicals.

Heterogeneous photocatalytic trifluoromethylation. In a typical procedure, a reaction mixture of heterocycle $1 \mathrm{a}(0.25 \mathrm{mmol})$, 2,4,6-collidine $(0.50 \mathrm{mmol})$, TfCl $(0.50 \mathrm{mmol})$, and $2.5 \mathrm{~mol} \% \mathrm{Zn}-$ TCTA (per mole organic dye ligand) in acetonitrile $(1 \mathrm{~mL})$ was subjected to visible-light irradiation from a $23 \mathrm{~W}$ household light bulb. An $84 \%$ yield of the desired product 2 a with the $\mathrm{CF}_{3}$ group positioned at the metabolically susceptible $\alpha$-position of the carbonyl group ${ }^{22}$ was obtained after $24 \mathrm{~h}$ (Figs. 3c, and 5, 2a). The control experiments indicated that light, an inert atmosphere, and $\mathrm{Zn}$-TCTA were indispensable for the reaction to proceed (Supplementary Table 2, entries 5-7). It should be noted that the addition of the radical scavenger tetramethylpiperidine- $N$-oxyl (TEMPO) to the reaction mixture inhibited the reaction (Supplementary Table 2, entry 13). For the reaction mixture with 2methyl-2-nitrosopropane dimer (MNP dimer, a typical $\mathrm{CF}_{3}$ radical scavenger) as a substitute for the substrate, a singlet-triplet splitting signal corresponding to the $\mathrm{CF}_{3}-\mathrm{MNP}$ • adduct was observed by in situ electron paramagnetic resonance $(\mathrm{EPR})$ spectroscopy $(g \text { value }=2.006)^{23}$ under visible-light irradiation. These results suggested the formation of $\mathrm{CF}_{3}$ radicals via 

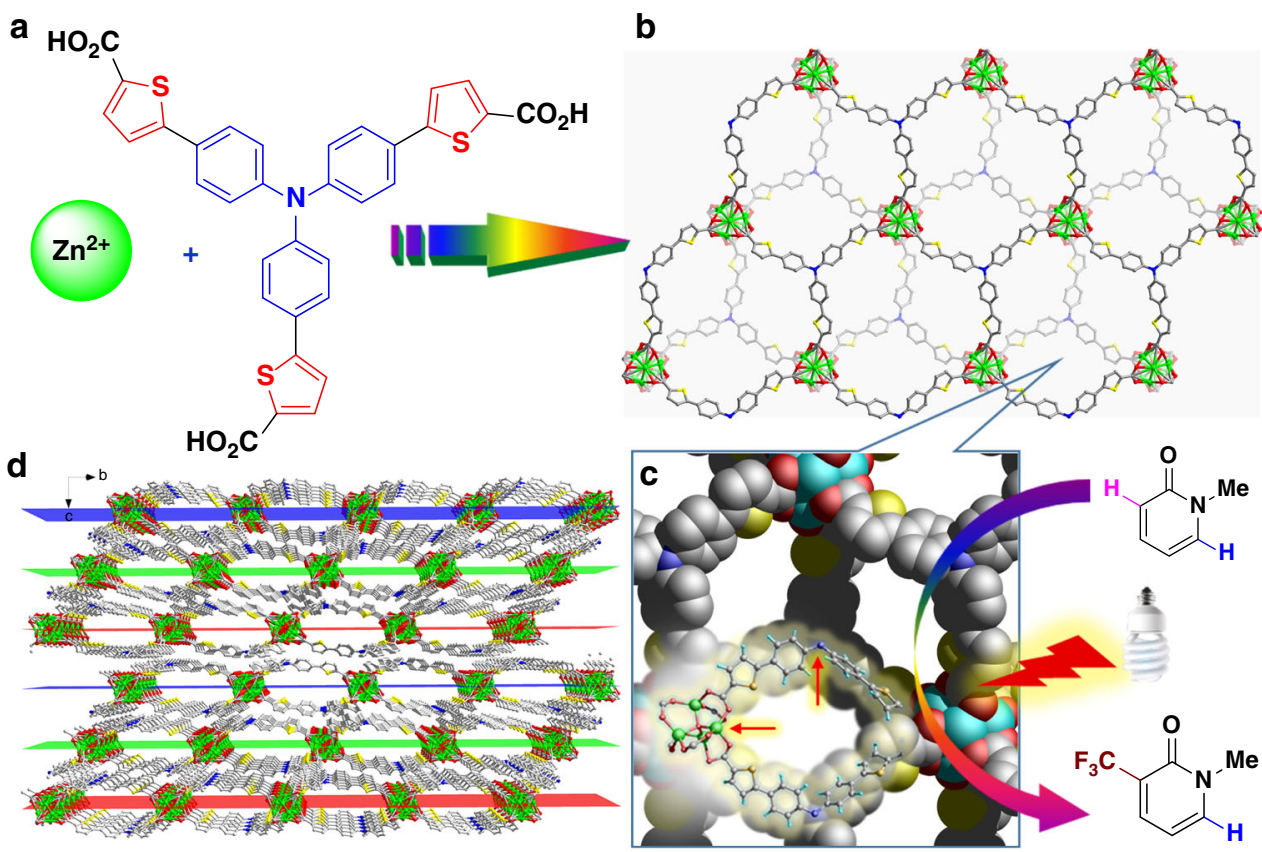

Fig. 1 Schematic illustration of the composition and structure of $\mathrm{Zn}$-TCTA. $\mathbf{a}$ The components. $\mathbf{b}$ The top view of the undulating monolayer with a virtual plane. c The intralayer cage showing the potential substrate binding site. d The packing pattern and open channels along the $a$ - or $b$-axis. Green, Zn; yellow, $\mathrm{S}$; red, O; blue, N; gray, C. Hydrogen atoms and solvent molecules are omitted for clarity
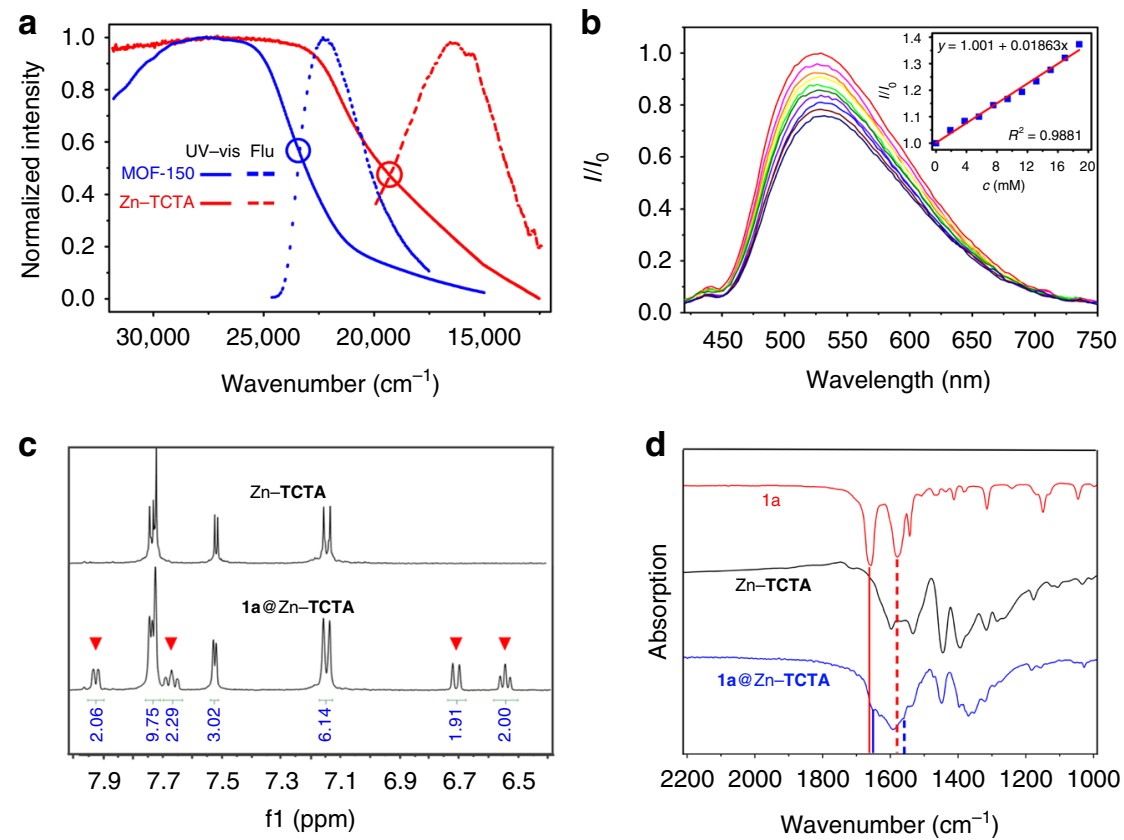

Fig. 2 The host-guest interactions between $\mathrm{Zn}-\mathbf{T C T A}$ and substrate/reagent. a Normalized absorption and emission spectra of $\mathrm{Zn}-\mathbf{T C T A}\left(\nu^{0-0}=19,274\right.$ $\left.\mathrm{cm}^{-1}, E^{0-0}=2.39 \mathrm{eV}\right)$ and MOF-150 $\left(\nu^{0-0}=23,468 \mathrm{~cm}^{-1}, E^{0-0}=2.91 \mathrm{eV}\right)$ excited at 390 and $350 \mathrm{~nm}$, respectively. b Fluorescence spectrum of ZnTCTA upon the addition of trifluoromethanesulfonyl chloride ( $\mathrm{TfCl}$ ) and the corresponding simulated Stern-Volmer curve (inset of $\mathbf{b}$ ) excited at $390 \mathrm{~nm}$; the intensity was recorded at $546 \mathrm{~nm}$. c ${ }^{1} \mathrm{H}$ NMR spectrum of the crystals of $\mathrm{Zn}-\mathbf{T C T A}$ and 1a@Zn-TCTA (digested in DMSO-d $6 / D C l$ ). Peaks marked with inverted red triangles represent the aromatic signals of the encapsulated substrate $\mathbf{1 a}$. $\mathbf{d}$ Comparison of the infrared (IR) spectrum of $\mathbf{1 a}$ (red line), $\mathrm{Zn}$ -TCTA (black line), and 1a@Zn-TCTA (blue line)

a photoinduced single electron transfer pathway (Supplementary Fig. 29). ${ }^{1} \mathrm{H}$ nuclear magnetic resonance (NMR) analysis of the digested crystals revealed that Zn-TCTA could adsorb approximately 2.0 equivalents (equiv) of $\mathbf{1 a}$ per unit (Fig. 2c). The infrared (IR) spectrum of the 1a-impregnated crystals showed the $\mathrm{C}=\mathrm{C}$ stretching vibrations of $1 \mathrm{a}$ at $1560 \mathrm{~cm}^{-1}$, indicating the fixation and possible activation of the alkene moiety within the pores of the coordination polymer (Fig. 2d).

Single-crystal X-ray structural analysis on the substrateimpregnated crystal1a@ Zn-TCTA revealed that the framework 
and pores of $\mathrm{Zn}$-TCTA were retained upon uptake of the substrate ${ }^{18,24}$, and indicated that 1a was fixed near the inner surface of the confined pores by coordination interactions between the unprotected zinc atom and the carbonyl oxygen of 1a $\left(\mathrm{O}_{1 \mathrm{a}} \cdots \mathrm{Zn}\right.$ distance of ca. $\left.2.20 \AA\right)$. The enforced close proximity between the photoactive TCTA $^{3-}$ unit and the metabolically susceptible $\alpha$-position of 1a (shortest interatomic distance of ca. $3.75 \AA$ for $\alpha$-position vs. ca. $4.11 \AA$ for $\delta$-position, respectively) provided the possibility to accelerate the reaction at the $\alpha$ position $^{16}$ (Figs. 3d, e and Supplementary Fig. 18). Because the oxidation of the $\mathrm{CF}_{3}$ radical adduct, which is known to be the rate-determining step ${ }^{6}$, could be accelerated by the closer spatial proximity in the case of $\mathrm{CF}_{3}$ radical addition at the $\alpha$-position of the carbonyl group, the specific spatial orientation of Zn-TCTA in the pores is expected to create the distinctive regioselectivity of trifluoromethylation in comparison to photocatalysis at the $\delta$ position by $f a c-\operatorname{Ir}(\text { Fppy })_{3}{ }^{4}$ (Fig. $3 \mathrm{~b}$ ). In this case, both the electronic and spatial effects are important to the heterogeneous reaction in the pores of $\mathrm{Zn}$-TCTA with microdynamics that are distinct from those of the homogeneous reaction.

After photocatalysis, Zn-TCTA was easily isolated from the reaction mixture by centrifugation, and the time-dependent conversion plots revealed that the photocatalyst could be reused at least three times without a remarkable decrease in reactivity (Fig. 4c and Supplementary Table 2, entries 10-12). The unchanged substrate ingress/egress ability of the recovered solids after three runs compared with that of the Zn-TCTA block crystals suggested that the capacity of the $\mathrm{Zn}$-TCTA pores was mainly retained (Supplementary Fig. 14). The X-ray powder diffraction pattern of the recovered catalyst indicated that the integrity of Zn-TCTA was basically maintained during the reaction. The remarkably decreased diffraction peak observed at a low angle possibly suggested the deterioration of long-range order in the vertical direction (Supplementary Fig. 25). It should also be noted that the micron-sized thin layers were exfoliated ${ }^{25,26}$ in situ from single-crystal blocks of the polymer on the basis of the scanning electron microscopy (SEM) (Supplementary Fig. 26) and transmission electron microscopy (TEM) (Fig. $4 \mathrm{~d}$ and Supplementary Fig. 27) images. Low-pressure $\mathrm{N}_{2}$ adsorption/ desorption of fresh $\mathrm{Zn}$-TCTA and the recycled sample were conducted at $77 \mathrm{~K}$. Both of fresh crystals of $\mathrm{Zn}$-TCTA and the recovered sample exhibited a Type-I sorption behaviour, with Brunauer-Emmett-Teller (BET) surface areas of 1383 and 1600 $\mathrm{m}^{2} \mathrm{~g}^{-1}$, respectively, which were calculated from $\mathrm{N}_{2}$ adsorption isotherms. Compared with the case of fresh Zn-TCTA, the mildly increased BET surface area of recovered sample might be attributed to the in situ exfoliation. The maintained pore size distributions reflected the stability of coordination polymer and the integrity of pores during photocatalysis (Supplementary Fig. 28).

Considering the unavoidable loss when recycling a small amount of catalyst after each run, a time-course experiment was performed using a large excess of $1 \mathbf{1 a}(2.5 \mathrm{mmol}, 10$ equiv). With the same quantity of $\mathrm{Zn}$-TCTA $(6.25 \mu \mathrm{mol}, 2.5 \mathrm{~mol} \%)$ and intermittent charging of the original amounts $(0.50 \mathrm{mmol}, 2.0$ equiv) of $\mathrm{TfCl}$ and the base additive each day, a final NMR yield of ca. $95 \%$ was obtained after ten consecutive catalytic runs without further derivatization of $\mathbf{2 a}$. It should be noted that the homogeneous control experiment exhibited remarkably destructive consumption of product 2a' when exposed to an excess of the reactive species over prolonged reaction times (Fig. 4b).

This approach can be applied to aromatic substrates bearing common heteroatomic functional groups that may act as drug candidates, enabling the photocatalytic reaction without the need for prefunctionalization of the arenes (Fig. 5). Gratifyingly, bioactive heteroarenes, such as derivatives of uracil (1b), caffeine (1d), theophylline (1e), and coumarin (1f), can be trifluoromethylated at metabolically susceptible positions with comparable yields. Note that this methodology was successfully extended to a series of fine chemicals or drug candidates with ambiguous aromatic reaction sites, such as the flavourant methylvanillin (11), the female hormone estrone derivative (1/m), and the nonsteroidal antiinflammatory drug derivatives of ibuprofen (1n) and indomethacin (10) with distinctive regioselectivity in comparison to the case using $f a c$-Ir(Fppy) ${ }_{3}$ as a photocatalyst (Supplementary Fig. 21). When substrate 1p, which has a molecular size larger than the cross-section of the open channel in the pores of $\mathrm{Zn}$ TCTA (Supplementary Fig. 16), was employed, a lower yield $(<10 \%)$ was detected $(2 \mathbf{p})$. Such a size-dependent transformation suggests that the catalytic reaction occurs within the pores of $\mathrm{Zn}$ TCTA, and the specific spatial orientation in the pores of $\mathrm{Zn}-$ TCTA can forge the improved and distinctive regioselectivity of trifluoromethylation compared with that obtained via homogeneous photocatalysis (Fig. $3 \mathrm{~b})^{4}$. Moreover, this heterogeneous route was amenable to either gram-scale preparation $\left(2 \mathbf{a}^{\dagger}\right)$ or the introduction of a different perfluoroalkyl group by using $\mathrm{C}_{4} \mathrm{~F}_{9} \mathrm{SO}_{2} \mathrm{Cl}$ (2aa) instead of $\mathrm{TfCl}$. In this case, our approach offers a potentially applicable route to effective protection of metabolically labile sites of late-stage drug intermediates via regioselective aryl trifluoromethyl functionalization.

Heterogeneous photocatalytic trifluoromethylation-arylation. By employing a series of $N$-aryl-methacrylamides and $N$-aroylmethacrylamides as substrates, we extended the photocatalytic approach to the selective conversion of unsaturated olefin ${ }^{27}$ moieties through tandem alkenyl trifluoromethylation-arylation (Fig. 6) ${ }^{28}$. This reaction involves an initial $\mathrm{CF}_{3}$ radical addition to an unsaturated alkene, intramolecular arylation of the $\mathrm{CF}_{3}$ radical adduct ${ }^{29,30}$, subsequent oxidation, and final deprotonation to afford biologically interesting 2,2,2-trifluoroethyl oxindoles ${ }^{31,32}$ and isoquinolinediones ${ }^{33}$ in considerable yields. When substrates bearing meta-substituted aryl moieties were used, only one type of regioisomer was obtained, indicating the high regioselectivities of the reactions $(\mathbf{4 c}-\mathbf{4 e})$. In addition to $\mathrm{CF}_{3}$, other perfluoroalkyl moieties, such as $\mathrm{C}_{4} \mathrm{~F}_{9}$, could also be introduced to the oxindole scaffold through this methodology (4aa).

Single-crystal structural analysis of 3a@Zn-TCTA suggested that the polymer structure was retained upon uptake of the substrate. Weak coordination interactions were also found between the metal node and carbonyl group of $\mathbf{3 a}(\mathrm{O} \cdots \mathrm{Zn}$ distance of ca. $3.76 \AA$ ), and $\mathrm{C}-\mathrm{H} \cdots \pi$ interactions were observed between the phenyl edge of TCTA ${ }^{3-}$ and phenyl $\pi$-plane of $3 \mathbf{a}$ (Fig. 7a and Supplementary Fig. 19). These interactions docked the substrates in confined spaces to facilitate tandem radical reactions. When no covalent anchoring positions are available on the substrate, the noncovalent substrate-catalyst interactions are critical for stereocontrol of the reaction; however, they are inherently labile toward thermal motions in solutions. Therefore, homogeneous stereoselective photoredox catalysis has long been hindered by the dearth of effective noncovalent binding strategies ${ }^{34-37}$. Inspired by previously described substrate-catalyst interaction patterns, we envisioned that multiple noncovalent interactions within a framework might induce a stereobias in $\mathrm{CF}_{3}$ radical transformations, increasing the molecular robustness towards oxidative metabolism of $\mathrm{sp}^{3} \mathrm{C}-\mathrm{H}$ bonds with stereospecificity.

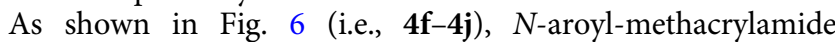
substrates bearing vicinal dialkyl-substituted unsaturated alkene moieties afforded products in good yields and diastereoselectivities as high as 95:5, which are superior to that obtained from a previously reported homogeneous approach ${ }^{38}$. Notably, 3j, 
a

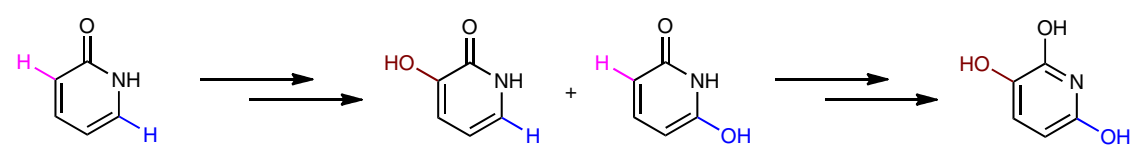

In vivo aryl oxidative metabolism

b

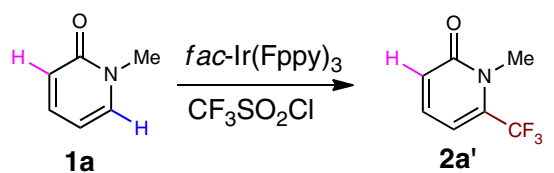

$1 \mathrm{a}$

$2 a^{\prime}$

State of the art of the homogeneous Photocatalytic trifluoromethylation

C

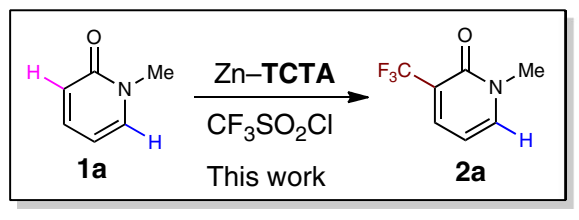

d

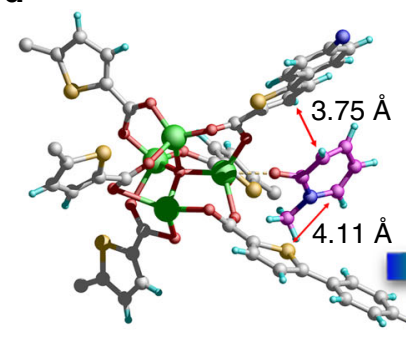

Substrate fixation \& activation e

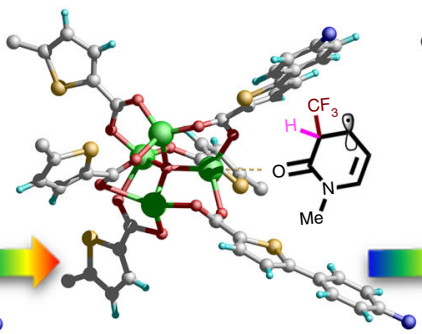

Oriented radical addition
Protection of metabolically susceptible positions of aromatics without prefunctionalization by photocatalytic trifluoromethyltion

Fig. 3 Schematic illustration of the photocatalytic site-specific trifluoromethylaion. a Potential reaction paths of the in vivo enzymatic aryl oxidative metabolism of $(2 \mathrm{H})$-pyridone. Complementary approaches to the protection of metabolically susceptible sites by trifluoromethylation using $\mathbf{b}$ fac-lr(Fppy) or $\mathbf{c} Z n-$ TCTA as the photocatalyst, respectively. d Crystal structure of 1a@Zn-TCTA showing the multiple interactions between 1a and the Zn-TCTA scaffold and the potential reaction paths $\mathbf{e}$ and $\mathbf{f}$ for the reaction

bearing a cyclic allyl moiety, which predominantly forms oligomers in the homogeneous case, was readily converted in a diastereocontrolled manner (d.r. of ca. 94:6) with an acceptable conversion in the presence of $\mathrm{Zn}$-TCTA.

A detailed structural analysis of a single crystal of the substrateenriched framework3f@Zn-TCTA indicated that within the confined environment, the unsaturated alkenyl and phenyl groups of $\mathbf{3 f}$ were folded in a face-to-face configuration by the $\mathrm{C}-\mathrm{H}$... O interactions between the node and $\mathrm{N}$-methyl group of $\mathbf{3 f}$ (C...O distance of ca. $3.46 \AA$ ) as well as by the $\mathrm{C}-\mathrm{H} \cdots \pi$ interactions between the phenyl edge of TCTA ${ }^{3-}$ and the phenyl $\pi$-system of $3 \mathbf{f}$. The proximity between the $\alpha$-carbon atom of the alkenyl group and the hydrogen atom in the ortho-position of the phenyl ring shielded the apparent $R e$-face of the alkenyl $\beta$-carbon, facilitating the formation of a properly oriented early transition state $^{39}$ to direct the tandem radical reactions in a highly diastereoselective manner (Figs. 7b, c and Supplementary Fig. 20).

\section{Discussion}

In conclusion, a new approach was developed for heterogeneous, efficient and site-specific trifluoromethylation of aromatic drug candidates without any directing groups. This approach included the insertion of thiophene moieties into the backbone of a TPAbased ligand to extend the light-harvesting ability and tune the photoelectronic properties. The enlarged $\pi$-system of the ligand favoured the interaction patterns to distort the coordination geometries of the metal nodes. The potential interactions of the metal nods docked the substrates near the photocatalytically active centres to directly activate the reaction sites of the substrates in special conformations. The comprehensive improvement in the electronic and spatial effects within the coordination polymer balanced the contradictory demands of the electronic effects and reaction dynamics, achieving regio- and diastereoselective discrimination between the reaction sites with unremarkable electronic/steric differences.

\section{Methods}

Materials and measurements. Unless otherwise stated, solvents were dried and distilled prior to use according to standard methods. 1,1',1" -[Nitrilotris(4,1-phenylenethiene-5,2-diyl)] triethanone, the precursor of $\mathrm{H}_{3} \mathbf{T C T A}^{40}$, and the starting materials $\mathbf{1} \mathbf{p}^{41}, \mathbf{3} \mathbf{a}$ and $\mathbf{3} \mathbf{c}^{42}, \mathbf{3} \mathbf{b}, \mathbf{3} \mathbf{d}$ to $3 \mathbf{i}^{43}$, and $3 \mathbf{j}^{38}$ were synthesized according to literature methods. The other substrates were commercially available and used as received. Thin-layer chromatography was carried out on $\mathrm{SiO}_{2}$ (silica gel 60 F254, Merck), and the spots were identified with UV light.

NMR spectrum were measured on a Bruker Avance $500 \mathrm{WB}$ and Bruker Avance $400 \mathrm{WB}$ spectrometer, and chemical shifts were recorded in parts per million (ppm, $\delta$ ). High-resolution mass spectrum (HRMS) were recorded on an liquid chromatography/quadrupole-time-of-flight mass spectrometer (Micromass, England) equipped with a Z-spray ionization source. Elemental analyses were performed on a Vario EL III elemental analyser. Inductively coupled plasma (ICP) was conducted on a NexION 300D spectrometer. Thermogravimetric analysis (TGA) was carried out with a Mettler-Toledo TGA/SDTA851 instrument.

Powder X-ray diffractogram measurements were performed with a PANalytical Empyrean X-ray powder diffractometer (Cu Ka radiation, $40 \mathrm{kV}, 40 \mathrm{~mA}$ ). Fourier Transform-IR spectra were recorded using $\mathrm{KBr}$ pellets on a JASCO FT/IR-430 instrument. The solid and liquid UV-vis spectra were recorded on a Hitachi U4100 UV-vis-NIR spectrophotometer and TU-1900 spectrophotometer, respectively. TEM images were collected on a Tecnai F30 microscope. SEM images were carried out over a Nova NanoSEM 450 microscope. 
a
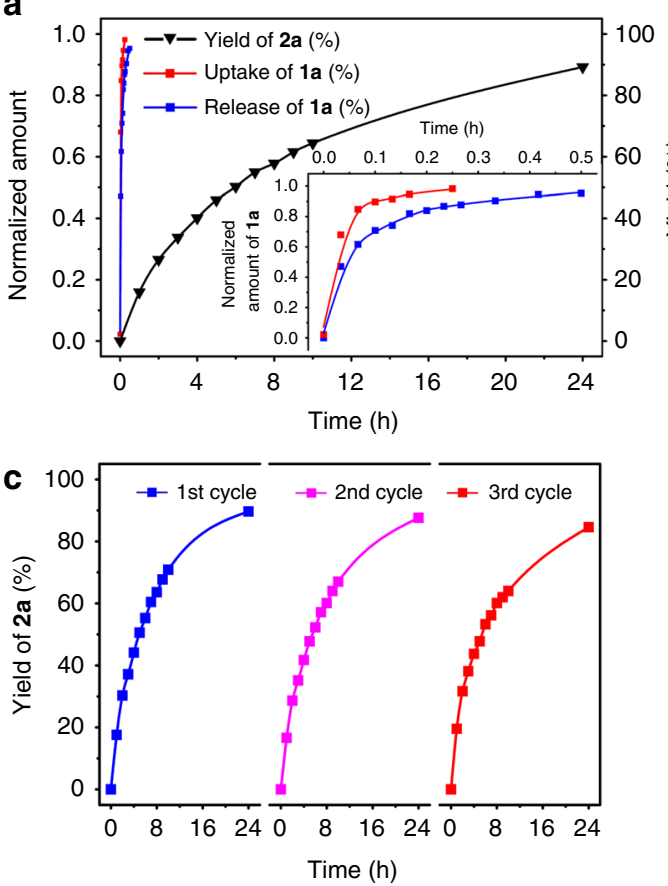

b
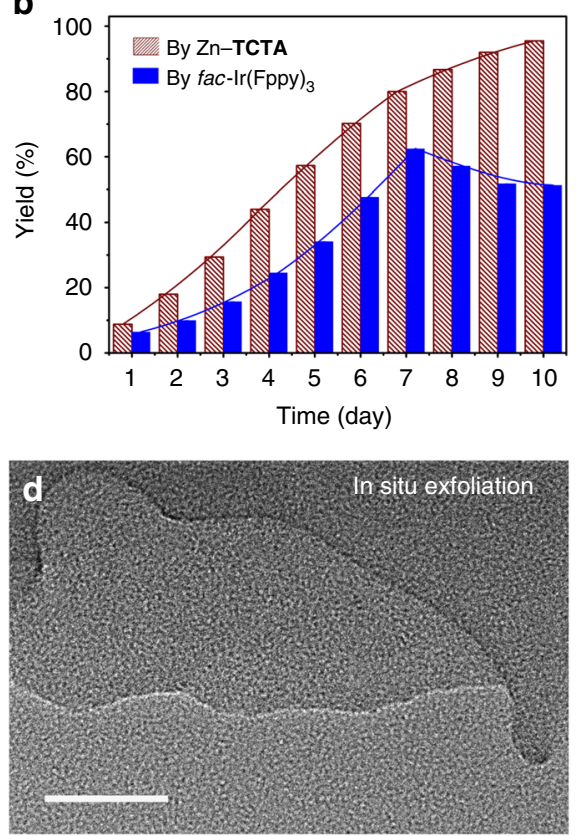

Fig. 4 Characterization of reaction dynamics and photocatalyst recyclability. a The kinetics of the ingress and egress of substrate 1a on Zn-TCTA block crystals in an acetonitrile suspension and the time-dependent conversion plots of the photocatalytic transformation with the insert showing the enlarged ingress/egress curves. $\mathbf{b}$ Histograms of the time-course reactions of excess $\mathbf{1 a}\left(2.5 \mathrm{mmol}, 10\right.$ equiv) in the presence of $\mathrm{Zn}-\mathbf{T C T A}$ and fac- $\mathrm{Ir}(\mathrm{Fppy})_{3}(6.25 \mu \mathrm{mol}, 2.5 \mathrm{~mol} \%)$ as photocatalysts, respectively. c Time-conversion plots of three rounds of the reaction using recycled catalyst Zn-TCTA. d A magnified TEM image of a crystalline powder of the photocatalyst Zn-TCTA after three rounds of reaction, showing the step-like cross-section of the laminated thin layers (inset, scale bar, 20 nm)

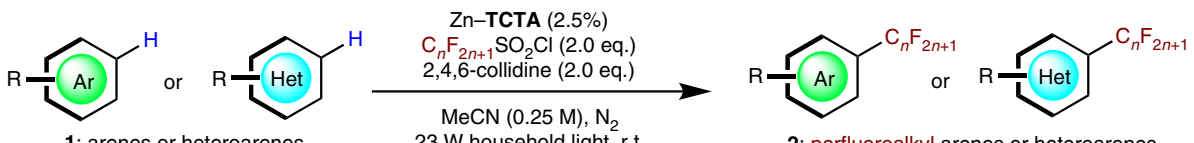

1: arenes or heteroarenes $\quad 23 \mathrm{~W}$ household light, r.t.

2: perfluoroalkyl arenes or heteroarenes
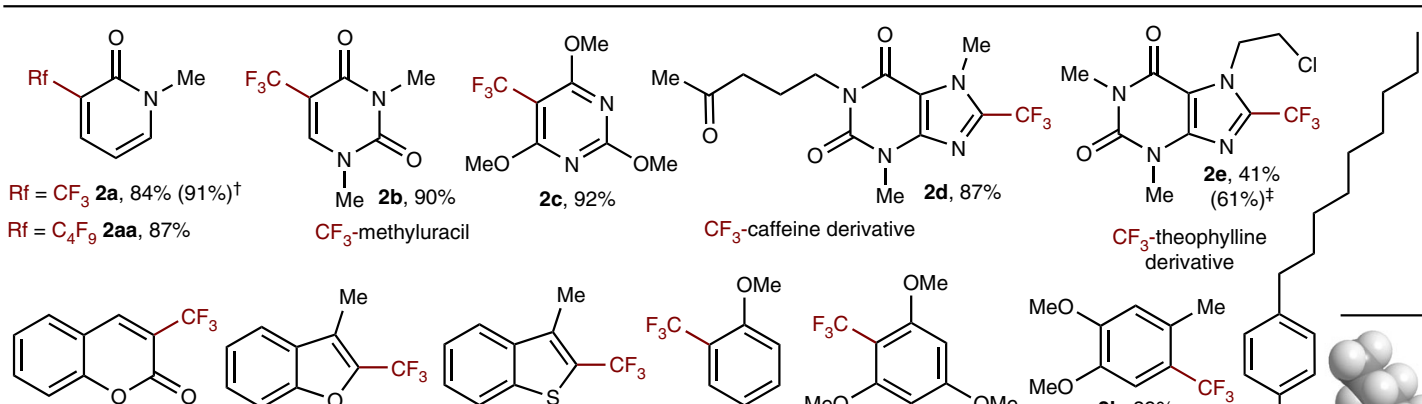<smiles>Cc1oc2ccccc2c1C</smiles><smiles>Cc1sc2ccccc2c1C</smiles>

2f, $62 \%$

2g, $76 \%$

2h, $72 \%$

2i, $77 \%$ OMe<smiles>COc1cc(OC)c(C(F)(F)F)c(OC)c1</smiles><smiles>CCc1ccc(Oc2ccc(C(C)(F)F)cc2)cc1C</smiles>
$\mathrm{CF}_{3}$-coumarin<smiles>COc1cc(C=O)cc(C(F)(F)F)c1OC</smiles>

2I, $79 \%(10: 1)^{*}$

2m, $65 \%(4: 1)^{\star}$

$\mathrm{CF}_{3}$-methylvanillin $\mathrm{CF}_{3}$-methylestrone

2j, $91 \%$<smiles>COc1ccc2c(c1)c(CC(=O)Cl)c(C)n2C(=O)c1ccc(Cl)cc1</smiles>

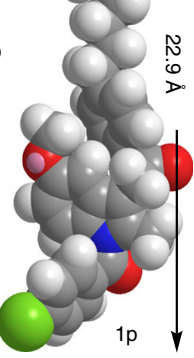

Fig. 5 Scope of photocatalytic trifluoromethylation of aromatics by Zn-TCTA. Reaction conditions: 1 ( 0.25 mmol, 1.0 equiv), RfCl (fluoroalkylation reagent as specified, 2.0 equiv), base additive (2.0 equiv), $\mathrm{Zn}$-TCTA ( 0.025 equiv), MeCN ( $1 \mathrm{~mL}$ ), $23 \mathrm{~W}$ household light, $\mathrm{N}_{2}$ atmosphere, room temperature, $24 \mathrm{~h}$. Isolated yields. $15 \mathrm{mmol}$-scale reaction. $\$ 3.0$ equiv of $\mathrm{TfCl}$ and collidine. ${ }^{*}$ Only major regioisomers are shown. The minor regioisomeric sites are labelled with their carbon atom numbers 

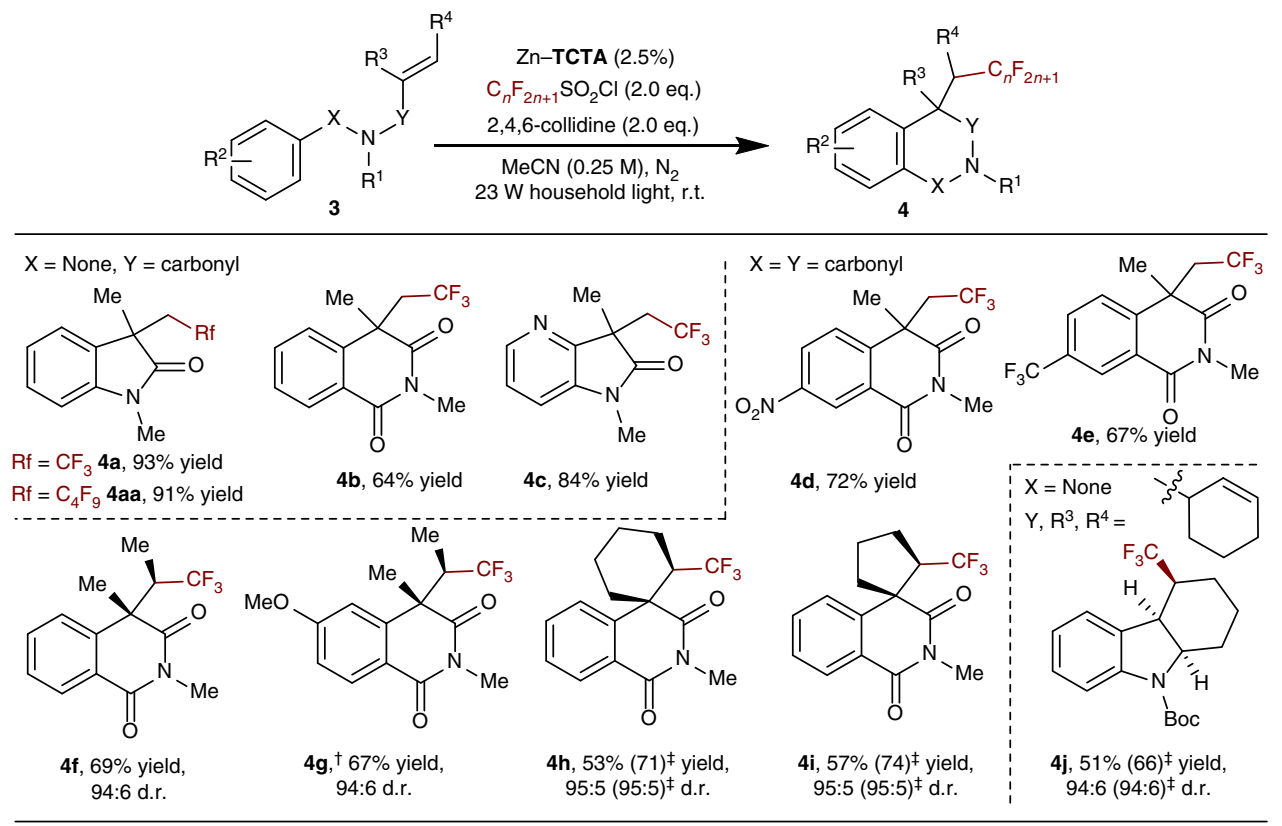

Fig. 6 Photocatalytic alkenyl trifluoromethylation-arylation by Zn-TCTA. Conditions were the same as those in Fig. 5 . Isolated yield. ${ }^{\dagger} .5$ or $\$ 3.0$ equiv of $\mathrm{TfCl}$ and collidine

a

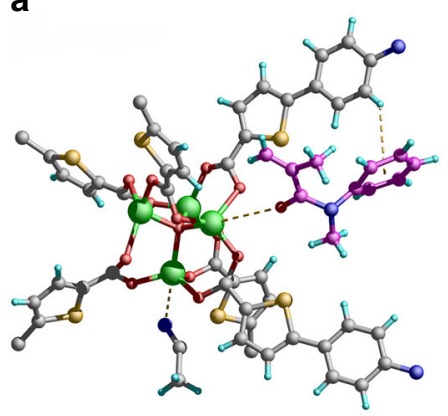

b

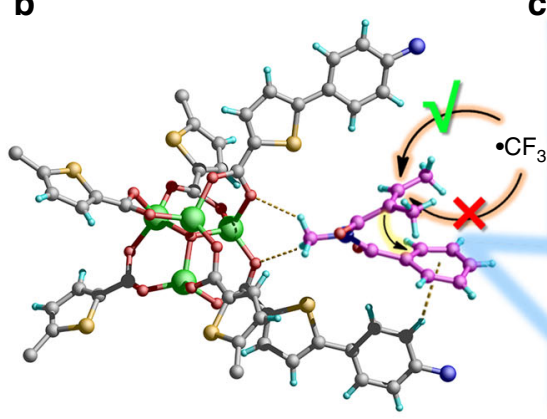

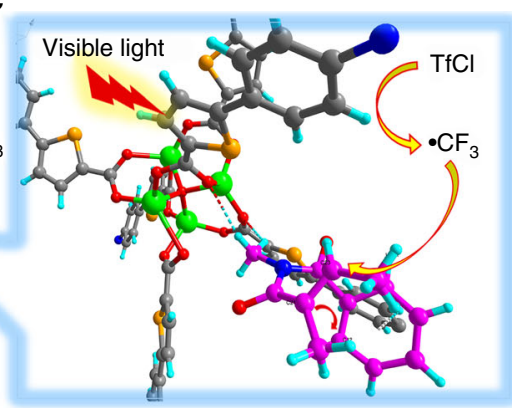

Fig. 7 Illustration of the interactions between the encapsulated substrates and Zn-TCTA. a Structure of 3a@Zn-TCTA. b Structure of 3f@Zn-TCTA. cThe plausible mechanistic interpretation of the role of substrate encapsulation in diastereocontrol

Solid-state cyclic voltammograms were measured using a carbon-paste working electrode; a well-ground mixture of each bulk sample and carbon paste (graphite and mineral oil) was set in the channel of a glass tube and connected to a copper wire. A platinum-wire counter electrode and an $\mathrm{Ag} / \mathrm{AgCl}$ reference electrode were used in an aqueous solution of $\mathrm{KNO}_{3}$. The solid fluorescent spectrum were measured on an Edinburgh FS920 instrument. The time-resolved luminescence spectrum were measured on an Edinburgh FLS920 spectrometer.

Single-crystal X-ray crystallography. Intensities were collected on a Bruker SMART APEX CCD diffractometer with graphite monochromated Mo-Ka radiation $(\lambda=0.71073 \AA)$ using the SMART and SAINT programs ${ }^{44,45}$. The structure was solved by direct methods and refined on F2 by full-matrix leastsquares methods with SHELXTL version $5.1^{46}$. Hydrogen atoms were fixed geometrically at calculated positions and allowed to ride on the parent nonhydrogen atoms. The SQUEEZE program was carried out for crystals $\mathrm{Zn}-$ TCTA, 1a@Zn-TCTA, and 3a@Zn-TCTA. Crystallographic data for ZnTCTA, 1a@Zn-TCTA,3a@Zn-TCTA, and 3f@Zn-TCTA are summarized in Supplementary Table 1. For all of the crystal data, one of the carboxylate oxygen atoms was disordered into two parts with the site occupancy factor (s.o.f.) of each part fixed at 0.5 .

In the refinement of crystal data of 1a@Zn-TCTA, 3a@Zn-TCTA, and 3f@ZnTCTA, to help the stability of the refinement for the impregnated related substrate molecules, the bond distances between several atoms were fixed; the geometrical constraints of idealized regular polygons were used for benzene rings, and thermal parameters on adjacent atoms in two molecules were restrained to be similar. The A alert error in the checklist for 3f@Zn-TCTA is due to the partial occupancy of the substrate molecules.
Synthesis of Zn-TCTA. A mixture of $\mathrm{H}_{3}$ TCTA ( $93 \mathrm{mg}, 0.15 \mathrm{mmol}$ ), $\mathrm{Zn}$ $\left(\mathrm{NO}_{3}\right)_{2} \cdot 6 \mathrm{H}_{2} \mathrm{O}(297 \mathrm{mg}, 1.0 \mathrm{mmol})$ was dissolved in $6 \mathrm{~mL}$ DMF in a Teflon-lined steel autoclave. The resulting mixture was kept in an oven at $100{ }^{\circ} \mathrm{C}$ for 3 days. The block red-brown crystals for X-ray structural analysis were collected by filtration, washed with acetonitrile, and then dried under vacuum. Yield: $70 \% .{ }^{1} \mathrm{H}$ NMR $(400$ MHz, DMSO- $\left.d_{6} / \mathrm{DCl}\right): \delta=7.72-7.70(\mathrm{~m}, 9 \mathrm{H}), 7.50(\mathrm{~d}, J=3.9 \mathrm{~Hz}, 3 \mathrm{H}), 7.13(\mathrm{~d}, J=$ $8.7 \mathrm{~Hz}, 3 \mathrm{H})$.

Dye uptake experiments. Crystals of Zn-TCTA were soaked in a saturated solution of methylene blue in acetonitrile for $12 \mathrm{~h}$, and the resulting crystals were washed with acetonitrile thoroughly until the solution became clear. The dried sample was dissociated by concentrated hydrochloric acid, and the resultant clear solution with a light olivine colour was diluted to $10 \mathrm{~mL}$ and adjusted to a $\mathrm{pH}$ of 1.5. The dye concentration was determined by comparing the solution UV-vis absorption with a standard curve of the dye.

Substrate ingress and egress experiments. Crystals of Zn-TCTA was soaked in a solution of substrate $1 \mathbf{a}$ in acetonitrile $(0.25 \mathrm{mmol} / \mathrm{mL})$, and the mixture was shaken by a vortex reactor. The uptake amount of $1 \mathbf{a}$ was monitored by timecourse sampling of supernatant and gas chromatography (GC). The previously obtained crystals saturated with substrate 1a was washed with a minimum amount of acetonitrile to remove the substrate absorbed on the surface, then immersed in acetonitrile, and shaken by a vortex reactor. The release amount of 1a was monitored time-coursely by GC analysis.

General procedure (GP) for photocatalysis by $\mathbf{Z n - T C T A}$. To a predried Pyrex tube equipped with a cooling water system, specified amounts of Zn-TCTA 
crystals $(0.00625 \mathrm{mmol})$ and substrate $(0.25 \mathrm{mmol})$ were added. After adding acetonitrile $(1 \mathrm{~mL}), 2,4,6$-collidine $(0.50 \mathrm{mmol})$, and $\mathrm{TfCl}(0.50 \mathrm{mmol})$ by syringe, the reaction mixture was stirred and illuminated with visible light by a $23 \mathrm{~W}$ household light under $\mathrm{N}_{2}$ atmosphere for $24 \mathrm{~h}$. The catalyst was recovered by centrifugation and filtration, and the filtrate was concentrated under reduced pressure. The product was isolated via flash chromatography on silica gel from the crude mixture.

Typical procedure for ten consecutive photocatalysis runs. To a predried Pyrex tube equipped with a cooling water system, specified amounts of photocatalyst $\mathrm{Zn}-$ TCTA $(0.00625 \mathrm{mmol})$ and substrate $\mathbf{1 a}(2.5 \mathrm{mmol})$ were added. After adding acetonitrile, $2,4,6$-collidine $(0.50 \mathrm{mmol})$ and $\mathrm{TfCl}(0.50 \mathrm{mmol})$ were added by syringe, and the reaction mixture was stirred and illuminated with visible light by a $23 \mathrm{~W}$ household light under $\mathrm{N}_{2}$ atmosphere for $24 \mathrm{~h}$. Then, another $0.5 \mathrm{mmol}$ of additive base and $\mathrm{TfCl}$ were added, and the reaction was intermittently charged with the previously mentioned reagents for a total of ten times. When $f a c-\operatorname{Ir}(\mathrm{Fppy})^{3}$ was used as the photocatalyst, the reaction was performed according to the literature protocol ${ }^{4}$, except for the use of ten equiv of $1 \mathrm{a}(2.5 \mathrm{mmol})$ and the intermittently charged additive base and $\mathrm{TfCl}$.

Substrate encapsulation experiments. The substrate-impregnated crystals were obtained by soaking crystals of Zn-TCTA in a solution of the substrate in acetonitrile ( $1 \mathrm{M})$ for $12 \mathrm{~h}$. After the soaked Zn-TCTA was washed with acetonitrile, the substrate-loaded crystals were directly used for single-crystal X-ray diffraction and IR or digested with DMSO- $d^{6} / \mathrm{DCl}$, and the amounts of released substrate molecules were quantified by ${ }^{1} \mathrm{H}$ NMR.

$\mathbf{N}_{\mathbf{2}}$ adsorption/desorption measurements. Low-pressure $\mathrm{N}_{2}$ sorption were conducted by using a Micrometritics ASAP 2020 measurements surface area and pore size analyzer up to saturated pressure at $77 \mathrm{~K}$. Before the $\mathrm{N}_{2}$ sorption measurements, the fresh crystals of $\mathrm{Zn}$-TCTA and the recovered sample were washed with acetonitrile and then subjected to vacuum heating.

\section{Data availability}

The X-ray crystallographic coordinates for the structures reported in this article have been deposited at the Cambridge Crystallographic Data Centre (CCDC) under the deposition numbers CCDC 1407818, 1546691, 1415189, and 1415190 (Supplementary Table 1). These data can be obtained free of charge from The Cambridge Crystallographic Data Centre via http://www.ccdc.cam.ac.uk/data_request/cif. All other data supporting the findings of this study are available within the article and its Supplementary Information files or from the corresponding author upon request.

Received: 2 January 2018 Accepted: 26 July 2018

Published online: 02 October 2018

\section{References}

1. Müller, K., Faeh, C. \& Diederich, F. Fluorine in pharmaceuticals: looking beyond intuition. Science 317, 1881-1886 (2007).

2. Furuya, T., Kamlet, A. S. \& Ritter, T. Catalysis for fluorination and trifluoromethylation. Nature 473, 470-477 (2011).

3. Studer, A. A. "Renaissance" in radical trifluoromethylation. Angew. Chem. Int. Ed. 51, 8950-8958 (2012).

4. Nagib, D. A. \& MacMillan, D. W. C. Trifluoromethylation of arenes and heteroarenes by means of photoredox catalysis. Nature 480, 224-228 (2011).

5. Liu, X., Xu, C., Wang, M. \& Liu, Q. Trifluoromethyltrimethylsilane: nucleophilic trifluoromethylation and beyond. Chem. Rev. 115, 683-730 (2015).

6. Charpentier, J., Früh, N. \& Togni, A. Electrophilic trifluoromethylation by use of hypervalent iodine reagents. Chem. Rev. 115, 650-682 (2015).

7. Alonso, C., De Marigorta, E. M., Rubiales, G. \& Palacios, F. Carbon trifluoromethylation reactions of hydrocarbon derivatives and heteroarenes. Chem. Rev. 115, 1847-1935 (2015).

8. Zhang, T. \& Lin, W. Metal-organic frameworks for artificial photosynthesis and photocatalysis. Chem. Soc. Rev. 43, 5982-5993 (2014).

9. Dhakshinamoorthy, A., Asiri, A. M. \& García, H. Metal-organic framework (MOF) compounds: photocatalysts for redox reactions and solar fuel production. Angew. Chem. Int. Ed. 55, 5414-5445 (2016).

10. Quah, H. S. et al. Multiphoton harvesting metal-organic frameworks. Nat. Commun. 6, 7954 (2015).

11. Mondloch, J. E. et al. Vapor-phase metalation by atomic layer deposition in a metal-organic framework. J. Am. Chem. Soc. 135, 10294-10297 (2013).

12. Zeng, L. et al. Organized aggregation makes insoluble perylene diimide efficient for the reduction of aryl halides via consecutive visible light-induced electron-transfer processes. J. Am. Chem. Soc. 138, 3958-3961 (2016).
13. Zhao, M., Ou, S. \& Wu, C.-D. Porous metal-organic frameworks for heterogeneous biomimetic catalysis. Acc. Chem. Res. 47, 1199-1207 (2014).

14. Chae, H. K., Kim, J., Friedrichs, O. D., Keeffe, M. O. \& Yaghi, O. M. Design of frameworks with mixed triangular and octahedral building blocks exemplified by the structure of $\left[\mathrm{Zn}_{4} \mathrm{O}(\mathrm{TCA})_{2}\right]$ having the pyrite topology. Angew. Chem. Int. Ed. 42, 3907-3909 (2003).

15. $\mathrm{Wu}, \mathrm{P}$. et al. Photoactive chiral metal-organic frameworks for light-driven asymmetric $\alpha$-alkylation of aldehydes. J. Am. Chem. Soc. 134, 14991-14999 (2012).

16. Xia, Z., He, C., Wang, X. \& Duan, C. Modifying electron transfer between photoredox and organocatalytic units via framework interpenetration for $\beta$ carbonyl functionalization. Nat. Commun. 8, 361 (2017).

17. Kawamichi, T., Haneda, T., Kawano, M. \& Fujita, M. X-ray observation of a transient hemiaminal trapped in a porous network. Nature 461, 633-635 (2009).

18. Inokuma, Y. et al. X-ray analysis on the nanogram to microgram scale using porous complexes. Nature 495, 461-466 (2013).

19. Grimsdale, A. C. \& Müllen, K. The chemistry of organic nanomaterials. Angew. Chem. Int. Ed. 44, 5592-5629 (2005).

20. Bétard, A. \& Fischer, R. A. Metal-organic framework thin films: from fundamentals to applications. Chem. Rev. 112, 1055-1083 (2012).

21. Lalevée, J. et al. Subtle ligand effects in oxidative photocatalysis with iridium complexes: application to photopolymerization. Chem. Eur. J. 17, 15027-15031 (2011)

22. Zefirov, N. S. et al. Degradation of pyridine by Arthrobacter crystallopoietes and Rhodococcus opocus strains. FEMS Microbiol. Lett. 118, 71-74 (1994).

23. Peng, Y. Y., Huang, J., Qu, Y. L. \& Zhao, C. X. ESR studies on the solvation of perfluoro-n-alkyl t-butyl nitroxides-a new scale for electronegativity of perfluoro-n-alkyl groups. Res. Chem. Intermed. 26, 587-597 (2000).

24. Jing, X., He, C., Dong, D., Yang, L. \& Duan, C. Homochiral crystallization of metal-organic silver frameworks: asymmetric [3+2] cycloaddition of an azomethine ylide. Angew. Chem. Int. Ed. 51, 10127-10131 (2012).

25. Peng, Y. et al. Metal-organic framework nanosheets as building blocks for molecular sieving membranes. Science 346, 1356-1359 (2014).

26. Junggeburth, S. C. et al. Ultrathin $2 \mathrm{D}$ coordination polymer nanosheets by surfactant-mediated synthesis. J. Am. Chem. Soc. 135, 6157-6164 (2013).

27. Yu, X. \& Cohen, S. M. Photocatalytic metal-organic frameworks for selective 2,2,2-trifluoroethylation of styrenes. J. Am. Chem. Soc. 138, 12320-12323 (2016).

28. Egami, H. \& Sodeoka, M. Trifluoromethylation of alkenes with concomitant introduction of additional functional groups. Angew. Chem. Int. Ed. 53, 8294-8308 (2014).

29. Wu, Z., Wang, D., Liu, Y., Huan, L. \& Zhu, C. Chemo- and regioselective distal heteroaryl ipso-migration: a general protocol for heteroarylation of unactivated alkenes. J. Am. Chem. Soc. 139, 1388-1391 (2017).

30. Xu, Y., Wu, Z., Jiang, J., Ke, Z. \& Zhu, C. Merging distal alkynyl migration and photoredox catalysis for radical trifluoromethylative alkynylation of unactivated olefins. Angew. Chem. Int. Ed. 56, 4545-4548 (2017).

31. Galliford, C. V. \& Scheidt, K. A. Pyrrolidinyl-spirooxindole natural products as inspirations for the development of potential therapeutic agents. Angew. Chem. Int. Ed. 46, 8748-8758 (2007).

32. Jimenez, J. I., Huber, U., Moore, R. E. \& Patterson, G. M. Oxidized welwitindolinones from terrestrial fischerella spp. J. Nat. Prod. 62, 569-572 (1999).

33. Vernekar, S. K. V. et al. Design, synthesis, biochemical, and antiviral evaluations of C6 benzyl and C6 biarylmethyl substituted 2hydroxylisoquinoline-1,3-diones: dual inhibition against HIV reverse transcriptase-associated RNase $\mathrm{H}$ and polymerase with antiviral activities. $J$. Med. Chem. 58, 651-664 (2015).

34. Inoue, Y. Asymmetric photochemical reactions in solution. Chem. Rev. 92, 741-770 (1992).

35. Nicewicz, D. A. \& MacMillan, D. W. C. Merging photoredox catalysis with organocatalysis: the direct asymmetric alkylation of aldehydes. Science $\mathbf{3 2 2}$, 77-80 (2008)

36. Gong, L., Wenzel, M. \& Meggers, E. Chiral-auxiliary-mediated asymmetric synthesis of ruthenium polypyridyl complexes. Acc. Chem. Res. 46, 2635-2644 (2011).

37. Du, J., Skubi, K. L., Schultz, D. M. \& Yoon, T. P. A dual-catalysis approach to enantioselective $[2+2]$ photocycloadditions using visible light. Science 344, 392-396 (2014).

38. Kong, W., Casimiro, M., Fuentes, N., Merino, E. \& Nevado, C. Metal-free aryltrifluoro methylation of activated alkenes. Angew. Chem. Int. Ed. 52, 13086-13090 (2013)

39. Porter, N. A., Giese, B. \& Curran, D. P. Acyclic stereochemical control in freeradical reactions. Acc. Chem. Res. 24, 296-304 (1991).

40. Min, J. et al. Effects of oligothiophene $\pi$-bridge length on physical and photovoltaic properties of star-shaped molecules for bulk heterojunction solar cells. J. Mater. Chem. A 2, 16135-16147 (2014). 
41. Kalgutkar, A. S., Marnett, A. B., Crews, B. C., Remmel, R. P. \& Marnett, L. J. Ester and amide derivatives of the nonsteroidal antiinflammatory drug, indomethacin, as selective Cy-clooxygenase-2 inhibitors. J. Med. Chem. 43, 2860-2870 (2000).

42. Pinto, A., Jia, Y., Neuville, L. \& Zhu, J. Palladium-catalyzed enantioselective domino Heck-cyanation sequence: development and application to the total synthesis of Esermethole and Physostigmine. Chem. Eur. J. 13, 961-967 (2007).

43. Millet, A. \& Baudoin, O. Palladium-catalyzed $\gamma$-selective arylation of zincated boc-allyl amines. Org. Lett. 16, 3998-4000 (2014).

44. SMART Data collection software (v5.629) (Bruker AXS Inc., Madison, WI, 2003).

45. SAINT, Data reduction software (v6.45) (Bruker AXS Inc., Madison, WI, 2003).

46. Sheldrick, G. M. SHELX-97, Program for Crystal Structure Analysis (University of Göttingen, Göttingen, 1997).

\section{Acknowledgements}

This work was supported by the National Natural Science Foundation of China (21402020, U1608224, and 21531001), the Fundamental Research Funds for the Central Universities (DUT16RC(4)09 and DUT18LK50), and the 111 Project (B16008).

\section{Author contributions}

T.Z. and X.G. contributed equally to this work. T.Z. and C.D. conceived the project, designed the experiments, and wrote the manuscript. T.Z., X.G. and Y.S. performed the experiments. C.H. and C.D. solved and refined the X-ray crystal structures. All authors discussed the results and commented on the manuscript.

\section{Additional information}

Supplementary Information accompanies this paper at https://doi.org/10.1038/s41467018-05919-6.

Competing interests: The authors declare no competing interests.

Reprints and permission information is available online at http://npg.nature.com/ reprintsandpermissions/

Publisher's note: Springer Nature remains neutral with regard to jurisdictional claims in published maps and institutional affiliations.

(c) Open Access This article is licensed under a Creative Commons Attribution 4.0 International License, which permits use, sharing, adaptation, distribution and reproduction in any medium or format, as long as you give appropriate credit to the original author(s) and the source, provide a link to the Creative Commons license, and indicate if changes were made. The images or other third party material in this article are included in the article's Creative Commons license, unless indicated otherwise in a credit line to the material. If material is not included in the article's Creative Commons license and your intended use is not permitted by statutory regulation or exceeds the permitted use, you will need to obtain permission directly from the copyright holder. To view a copy of this license, visit http://creativecommons.org/ licenses/by/4.0/.

(C) The Author(s) 2018 